\title{
Saúde: vulnerabilidade social, vizinhança e atividade física
}

\author{
Health: social vulnerability, \\ neighborhood and physical activity
}

Manoel Carlos Sampaio de Almeida Ribeiro
Rita Barradas Barata

\section{Resumo}

Este artigo analisa a associação entre atividade física, grau de vulnerabilidade social e vizinhança na área central de São Paulo. Foram estudados os níveis de atividade física de adultos moradores em setores censitários vulneráveis e não vulneráveis relacionando esse comportamento com o contexto do bairro e com características sociodemográficas dos indivíduos. A distribuição dos níveis de atividade física foi diferente para os dois grupos, predominando, no grupo vulnerável, as pessoas ativas no trabalho sem relação com as condições de vizinhança. Já no grupo não vulnerável a atividade física estava dividida entre lazer e trabalho, e a maioria é pouco ativa. Nesse grupo há associação entre atividade física e gênero, não ter carro e sensação de segurança à noite no próprio bairro.

Palavras-chave: vulnerabilidade social; vizinhança; atividade física; saúde urbana.

\begin{abstract}
The article examines the association between physical activity, social vulnerability level and neighborhood in São Paulo's downtown. Physical activity levels were studied in adults living in vulnerable and non-vulnerable census tracts, and physical activity practice was related to neighborhood characteristics and to the individuals' sociodemographic variables. The distribution of physical activity levels was different for the two groups: in the vulnerable group, active people at the workplace predominated, unrelated to neighborhood conditions. In the non-vulnerable group, physical activity is divided between leisure and work, and most are somewhat active. In this group, there is an association between physical activity and gender, not having a car and sense of security at night in one's own neighborhood.
\end{abstract}

Keywords: social vulnerability; neighborhood; physical activity; urban health 


\section{Introdução}

0 padrão de urbanização acelerado imprimiu às grandes metrópoles periféricas algumas características comuns, tais como a precariedade da expansão da área urbana e a transformação permanente dos espaços intraurbanos geralmente marcados por processos de segregação socioespacial e por impactos negativos sobre 0 meio ambiente.

As décadas de 1980 e 1990 modificaram substancialmente a região metropolitana da Grande São Paulo. De uma região basicamente industrial até a década de 1970, ela passou a ser um polo de atividades concentradas no setor terciário e quaternário da economia, alterando o processo de uso do solo e a divisão socioespacial do trabalho, que no período anterior havia determinado a ampliação da periferia da cidade e a formação de anéis concêntricos em torno do centro expandido (Bousquat e Nascimento, 2001).

Durante todo o século $X X$, a história da urbanização de São Paulo foi marcada por um processo de periferização da pobreza e pela criação e abandono de diversas centralidades (Iglesias, 2002). Na primeira metade do século $\mathrm{XX}$, a urbanização da cidade separou as elites econômicas, que ficavam nas chamadas partes altas da cidade (Campos Elíseos, Higienópolis, Avenida Paulista), e alguns segmentos profissionais, que ficavam nos bairros operários habitados predominantemente por imigrantes europeus (Brás, Bom Retiro, Barra Funda, Belém, Mooca, Pari). A partir da década de 1960, a expansão das periferias pobres se deu em todas as direções com maior adensamento nas zonas sul e leste do município, onde se concentraram as grandes levas de migrantes nacionais chegados para trabalhar na indústria automotora e de bens duráveis. Progressivamente, com as transformações econômicas, novos "centros" foram se delineando na cidade, abandonando o centro histórico que passou por grandes transformações (Iglesias, 2002).

Como as outras grandes metrópoles mundiais, São Paulo enfrenta os problemas decorrentes dessas transformações econômicas que resultaram em profunda modificação do mercado de trabalho, gerando desemprego estrutural de parcela considerável dos trabalhadores não qualificados e crescimento das relações informais e da precarização das condições de trabalho. Territorialmente, esses processos se refletem em segmentação e diferenciação dos espaços urbanos com desigualdades marcantes no provimento dos serviços essenciais, tais como saneamento, limpeza urbana, transporte viário, habitação e urbanismo. 0 cenário urbano é fortemente marcado pelos contrastes sociais e pelas desigualdades entre a extrema riqueza e a extrema pobreza, geralmente segregadas espacialmente, mas em certas situações, como no distrito do Morumbi, convivendo lado a lado (Barata; Ribeiro e Cassanti, 2011).

A região central de São Paulo, constituída por 11 dos 97 distritos que compõem a cidade, é um exemplo típico de território marcado por extrema heterogeneidade no qual coexistem diferentes grupos sociais com perfis demográficos e socioeconômicos bastante diversos. Alguns bairros, como Higienópolis, ainda conservam parte da população mais rica da cidade, enquanto outros, como o Brás e o Bom Retiro, sofreram profundas transformações com a presença cada vez maior de migrantes estrangeiros. A área central concentra grande parte dos moradores em situação de rua, que 
estão entre os grupos mais marginalizados da cidade (Prefeitura do Município de São Paulo, 2001). Na última década, a população em situação de rua cresceu $65 \%$, e $55 \%$ dela se concentra na região central da cidade (Fundação Seade, 2015).

Os distritos que constituem a zona central reúnem 4,25\% da população de São Paulo, cerca de 492 mil habitantes, população equivalente à de um município de grande porte. A população é estacionária, com 14,5\% de idosos e $14,5 \%$ de jovens (ibid.). A renda per capita média é muito variável entre os distritos. Quatro deles apresentam valores inferiores à média municipal, e os demais apresentam renda per capita variando entre 1,36 e 3,36 vezes a média municipal, em valores nominais de 2010 (ibid.).

Figura 1 - Mapa dos distritos administrativos da área central do município de São Paulo

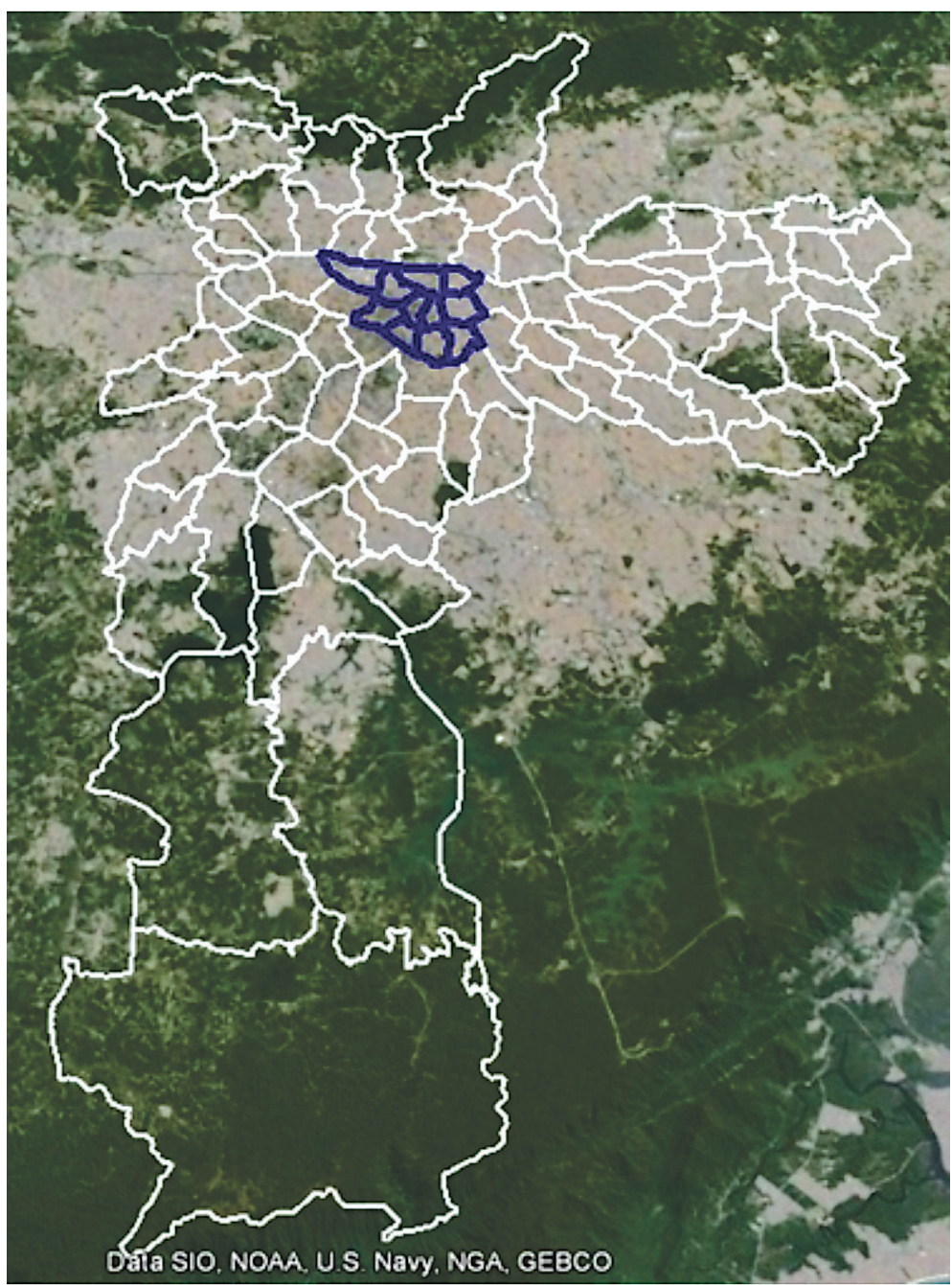


A saúde é resultante de um processo multidimensional envolvendo condições de vida e contextos sociais nos quais os grupos sociais vivem (World Health Organization, 2007). O perfil epidemiológico, ou seja, as necessidades de saúde dos diferentes grupos sociais, depende de um conjunto amplo de determinantes estruturais e intermediários, além das características individuais. Dentre os determinantes estruturais, merece destaque 0 contexto socioeconômico e político definido pelas condições de governança; políticas macroeconômicas; políticas sociais relacionadas ao mercado de trabalho, moradia e ocupação do solo urbano; políticas públicas relacionadas a educação, saúde e proteção social e os valores culturais da sociedade. Esse contexto, por sua vez, determina a estrutura de classes sociais, as relações de gênero e etnia e a estratificação por educação, ocupação e renda. Os determinantes intermediários referem-se às situações materiais de vida, os comportamentos e os fatores biológicos e psicossociais que podem favorecer a manutenção da saúde ou facilitar o surgimento das doenças. Os contextos de vulnerabilidade social correspondem a esse nível intermediário de determinação. 0 próprio sistema de saúde é considerado um dos determinantes intermediários, uma vez que sua forma de organização e as ações que ele desenvolve podem contribuir para reduzir ou para intensificar as desvantagens produzidas pelos determinantes estruturais. As condições e características de vizinhança também fazem parte dos determinantes intermediários, podendo afetar vários comportamentos relacionados com a saúde.

\section{Vulnerabilidade social: moradores da área central}

As condições de vida dos grupos humanos que vivem na área central da cidade de São Paulo impactam o estado de saúde e acabam por determinar, através dos processos de reprodução social, situações que favorecem a ocorrência de doenças e problemas de saúde, mas também situações que podem favorecer a manutenção da saúde, em um processo contraditório de elementos positivos e negativos, que acaba por conformar o perfil epidemiológico dos diferentes grupos (Castellanos, 1997 e Paim, 1997).

0 índice Paulista de Vulnerabilidade Social - IPVS construído a partir da composição de variáveis socioeconômicas e variáveis demográficas permite a estratificação dos setores censitários em seis diferentes grupos, desde aqueles com baixíssima vulnerabilidade social até aqueles com vulnerabilidade social muito alta, possibilitando, assim, uma ferramenta para a análise de diferentes grupos de moradores segundo sua vulnerabilidade social (Fundação Seade, 2010).

Para contrastar diferentes situações sociais, os autores realizaram inquérito domiciliar em amostra de moradores adultos dos 356 setores censitários classificados como de baixíssima vulnerabilidade social e em amostra de moradores dos 48 setores censitários classificados entre aqueles com vulnerabilidade média, alta e muito alta.

0 cálculo inicial da amostra previa a realização de 500 entrevistas em cada estrato. 
A partir da listagem dos setores censitários, foram sorteados 25 setores em cada estrato, utilizando um procedimento sistemático com partilha proporcional ao tamanho da população. Os dados dos setores censitários foram obtidos do censo de 2000. Dessa forma, avaliou-se necessário realizar um arrolamento para a atualização de todos os domicílios dos setores censitários sorteados.

0 arrolamento permitiu compor uma listagem com todos os 16.872 endereços dos domicílios de cada setor. A partir dessa listagem, procedeu-se a um sorteio sistemático de 40 domicílios ( $20+20$ reservas). Os 20 domicílios reservas eram utilizados quando não se conseguia completar a amostra com os 20 domicílios, em função de recusa ou após três visitas malsucedidas.

Foi entrevistado um indivíduo por domicílio, sorteado a partir da enumeração dos moradores elegíveis daquele domicílio. Dessa forma, foram realizadas 917 entrevistas (92\% do previsto), sendo 428 entrevistas (86\%) no estrato de setores censitários sem vulnerabilidade social pelo IPVS e 487 (97\%) no estrato com setores com vulnerabilidade social.

Tabela 1 - Características sociodemográficas dos grupos de moradores em setores censitários sem ou com vulnerabilidade social, centro da cidade de São Paulo, 2008

\begin{tabular}{|c|c|c|}
\hline Característica & $\begin{array}{c}\text { Grupo sem vulnerabilidade } \\
\text { (\% e IC } 95 \%)\end{array}$ & $\begin{array}{c}\text { Grupo com vulnerabilidade } \\
\text { (\% e IC } 95 \%)\end{array}$ \\
\hline $\begin{array}{l}\text { Idade } \\
\text { Jovem } \\
\text { Adulto jovem } \\
\text { Adulto } \\
\text { Idoso }\end{array}$ & $\begin{array}{r}10,2(7,6-13,4) \\
35,6(31,1-40,2) \\
33,0(28,7-37,5) \\
21,2(17,5-252)\end{array}$ & $\begin{array}{r}17,1(14,5-21,2) \\
44,1(39,8-48,7) \\
29,2(25,3-33,2) \\
9,0(6,7-118)\end{array}$ \\
\hline $\begin{array}{l}\text { Sexo } \\
\text { Masculino } \\
\text { Feminino }\end{array}$ & $\begin{array}{l}54,0(49,2-58,6) \\
46,0(41,4-50,8)\end{array}$ & $\begin{array}{l}57,1(52,7-61,4) \\
42,9(38,6-47,4)\end{array}$ \\
\hline $\begin{array}{l}\text { Escolaridade } \\
\text { Sem escolaridade } \\
\text { Ensino fundamental } \\
\text { Ensino médio } \\
\text { Ensino superior }\end{array}$ & $\begin{array}{r}0,9(0,3-2,2) \\
16,6(13,2-20,4) \\
20,8(17,1-24,7) \\
61,7(56,7-65,9)\end{array}$ & $\begin{array}{r}5,7(3,9-8,1) \\
52,0(47,5-56,5) \\
30,4(26,4-34,6) \\
11,9(9,3-15,0)\end{array}$ \\
\hline $\begin{array}{l}\text { Classes de consumo } \\
\text { E } \\
\text { D } \\
\text { C } \\
\text { B } \\
\text { A }\end{array}$ & $\begin{array}{r}4,4(2,7-6,7) \\
43,6(38,6-48,0) \\
17,8(14,3-21,5) \\
21,1(17,3-25,0) \\
13,1(10,1-16,5)\end{array}$ & $\begin{array}{r}24,1(20,2-27,8) \\
66,6(61,6-70,1) \\
6,4(4,4-8,8) \\
2,3(1,2-3,9) \\
0,6(0,2-1,6)\end{array}$ \\
\hline $\begin{array}{l}\text { Renda familiar (SM) } \\
\text { Até } 1 \\
1-2 \\
3-5 \\
6-10 \\
11 \text { ou mais }\end{array}$ & $\begin{array}{r}1,7(0,7-3,2) \\
13,7(10,1-16,5) \\
18,8(14,5-21,8) \\
29,8(24,3-32,8) \\
36,1(30,0-39,0)\end{array}$ & $\begin{array}{r}12,4(9,1-14,8) \\
57,3(49,6-58,4) \\
19,6(15,2-22,1) \\
8,3(5,7-10,5) \\
2,4(1,2-3,9)\end{array}$ \\
\hline
\end{tabular}


0 grupo vulnerável era composto predominantemente por indivíduos jovens e adultos jovens, sem escolaridade ou com ensino fundamental, pertencentes aos estratos $\mathrm{D}$ e $\mathrm{E}$ das classes de consumo, com renda familiar inferior a três salários mínimos.

0 grupo sem vulnerabilidade social era composto predominantemente por indivíduos adultos e idosos, com escolaridade de nível superior, pertencentes às classes de consumo $A, B$ ou $C$, com renda familiar superior a cinco salários mínimos.

As condições de vizinhança constituem outro dos determinantes intermediários e referem-se ao entorno mais imediato no qual as atividades da vida cotidiana se desenvolvem. $A$ partir de 2007-2008, pela primeira vez na história, metade da população mundial tornou-se urbana e, embora a maioria do mundo desenvolvido tenha se tornado altamente urbanizado há bastante tempo, as transformações mais rápidas e numericamente importantes ocorreram, na última década, nos países de renda média ou baixa (Vlahov, 2015).

Esse processo acelerado de urbanização, de forma desordenada e sem que a infraestrutura urbana fosse capaz de acompanhá-lo representa um enorme desafio principalmente para os grupos socialmente mais vulneráveis, em áreas em que as desvantagens ambientais, sociais e econômicas de encontram concentradas (ibid.).

As desigualdades sociais apresentadas pelos distintos grupos urbanos evidenciam-se também como desigualdades espaciais entre os diferentes bairros e áreas de vizinhança, refletindo, em seu perfil epidemiológico, não apenas as diferenças de exposição a agentes físico, químicos e biológicos, mas também os comportamentos e os estilos de vida condicionados pela inserção social e pelo contexto local que podem ou não favorecer a prática de comportamentos saudáveis.

\section{Vizinhança: contextos urbanos da vida cotidiana}

Vizinhança é um espaço geográfico demarcado no qual os moradores compartilham as condições de vida cotidiana (Boclin; Faerstein e Leon, 2014). 0 ambiente ou espaço construído refere-se às qualidades estéticas, físicas e funcionais dos bairros ou da vizinhança em que as pessoas vivem, incluindo o padrão de uso da terra, o padrão de construções e o arruamento que juntos podem oferecer oportunidades ou colocar obstáculos para o deslocamento ativo das pessoas e para a prática de atividades físicas, além de acesso a alimentação saudável, espaços de lazer e oferta de bens e serviços (King e Clarke, 2015).

Além das características mencionadas, outros aspectos da vizinhança, tais como sensação de segurança para pedestres, ausência de delitos, existência de edificações degradadas e pichações, a confiança nos vizinhos também contribuipara gerar um ambiente mais ou menos saudável nas áreas urbanas (Jongennel-Grimen et al., 2014).

Em pesquisas empíricas, a vizinhança ou ambiente construído tem sido operacionalizado através de seis dimensões: densidade residencial, conectividade, acessibilidade a serviços e destinos, facilidades para caminhada e ciclismo, qualidade estética e segurança (Meng et al., 2014). Os mecanismos através dos quais a vizinhança pode influenciar o perfil epidemiológico 
dos moradores podem incluir processos de mediação, tais como estilo de vida, comportamentos saudáveis, oportunidade para realizar atividades físicas, acesso a alimentos saudáveis e a serviços de saúde, além de efeitos das normas sociais locais ou de efeitos cumulativos do stress crônico (Boone-Heinonen et al., 2011)
A área central da cidade de São Paulo é bastante heterogênea quanto às características de vizinhança. Os setores censitários classificados nos estratos de média, alta ou muito alta vulnerabilidade social apresentam características muito distintas daqueles classificados no estrato de baixíssima vulnerabilidade social.

Tabela 2 - Aspectos de vizinhança dos grupos sem ou com vulnerabilidade social no centro da cidade de São Paulo, 2008

\begin{tabular}{|c|c|c|}
\hline Aspectos & $\begin{array}{c}\text { Grupo sem vulnerabilidade } \\
\text { (\% e IC } 95 \%)\end{array}$ & $\begin{array}{c}\text { Grupo com vulnerabilidade } \\
\text { (\% e IC } 95 \%)\end{array}$ \\
\hline $\begin{array}{l}\text { Satisfação com o bairro } \\
\text { Insatisfeito } \\
\text { Satisfeito } \\
\text { Muito satisfeito }\end{array}$ & $\begin{array}{r}1,4(0,5-2,8) \\
73,7(69,4-77,7) \\
24,9(20,9-29,1)\end{array}$ & $\begin{array}{r}18,4(15,1-22,0) \\
70,5(66,2-74,3) \\
11,2(8,5-14,2)\end{array}$ \\
\hline $\begin{array}{l}\text { Ruas pouco iluminadas } \\
\text { Não } \\
\text { Sim }\end{array}$ & $\begin{array}{l}74,1(69,8-78,1) \\
25,9(21,9-30,2)\end{array}$ & $\begin{array}{l}43,3(38,9-47,7) \\
56,7(52,3-61,1)\end{array}$ \\
\hline $\begin{array}{l}\text { Há muito barulho } \\
\text { Não } \\
\text { Sim }\end{array}$ & $\begin{array}{l}35,3(30,9-40,0) \\
64,7(60,0-69,1)\end{array}$ & $\begin{array}{l}43,6(39,3-48,1) \\
56,4(51,9-60,7)\end{array}$ \\
\hline $\begin{array}{l}\text { Há poluição ambiental } \\
\text { Não } \\
\text { Sim }\end{array}$ & $\begin{array}{l}15,1(11,9-18,7) \\
84,9(81,3-88,0)\end{array}$ & $\begin{array}{l}13,2(10,4-16,4) \\
86,8(83,5-89,6)\end{array}$ \\
\hline $\begin{array}{l}\text { Há lixo e entulho nas ruas } \\
\text { Não } \\
\text { Sim }\end{array}$ & $\begin{array}{l}60,1(55,4-64,7) \\
39,9(35,3-44,5) \\
\end{array}$ & $\begin{array}{r}25,3(21,5-29,3) \\
74,7(70,6-78,4) \\
\end{array}$ \\
\hline $\begin{array}{l}\text { Faltam espaços verdes } \\
\text { Não } \\
\text { Sim }\end{array}$ & $\begin{array}{l}48,1(43,4-52,8) \\
51,9(47,1-56,5)\end{array}$ & $\begin{array}{l}28,2(24,3-32,3) \\
71,8(67,6-75,6)\end{array}$ \\
\hline $\begin{array}{l}\text { Há risco para pedestres e/ou ciclistas } \\
\text { Não } \\
\text { Sim }\end{array}$ & $\begin{array}{l}13,6(10,5-17,0) \\
86,4(82,8-89,5)\end{array}$ & $\begin{array}{l}24,1(20,4-28,0) \\
75,9(78,5-85,3)\end{array}$ \\
\hline $\begin{array}{l}\text { Caminhar à noite dá sensação de: } \\
\text { Muita segurança } \\
\text { Segurança } \\
\text { Insegurança }\end{array}$ & $\begin{array}{r}4,7(2,9-7,0) \\
17,3(13,9-21,1) \\
78,0(73,9-81,7)\end{array}$ & $\begin{array}{r}4,1(2,6-6,2) \\
21,3(17,8-25,1) \\
74,5(70,5-78,2)\end{array}$ \\
\hline $\begin{array}{l}\text { Medo de assalto } \\
\text { Todo o tempo } \\
\text { Frequentemente } \\
\text { Nunca }\end{array}$ & $\begin{array}{r}5,8(3,8-8,3) \\
68,3(63,7-72,5) \\
25,9(21,9-30,1)\end{array}$ & $\begin{array}{l}20,5(17,2-24,3) \\
44,4(39,9-48,7) \\
35,1(30,9-39,4)\end{array}$ \\
\hline
\end{tabular}


0 grupo com vulnerabilidade social avaliou sua vizinhança como pouco iluminada, mais barulhenta, com presença de lixo e entuIho nas ruas, sem espaços verdes como parques e jardins, com maior risco para pedestres e ciclistas, insegura à noite, e maior proporção de pessoas relatou ter medo de assalto o tempo todo, quando comparado com o grupo sem vulnerabilidade social. Mesmo assim os moradores referiram estarem satisfeitos com o bairro em que vivem, embora maior proporção, em comparação ao grupo sem vulnerabilidade, referiu estar insatisfeita.

\section{Vizinhança sem vulnerabilidade}

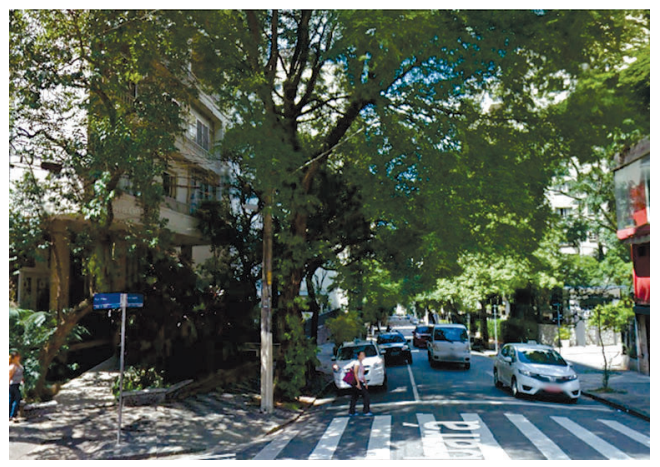

As vizinhanças nestas áreas não vulneráveis tende a ser arborizadas com calçadas e ruas limpas e em bom estado.

No geral são áreas residenciais com comércio e serviços locais.

Não há lixo na rua e as edificações estão em bom estado.

Moradores em situação de rua são ocasionalmente encontrados nesta vizinhança.

Pichações ocasionais compõem uma paisagem urbana, estruturada que passa uma sensação de segurança.
Vizinhanças mais degradadas são menos atrativas para pessoas com renda mais alta, levando, assim, a um processo de segregação espacial no qual as pessoas com melhores condições socioeconômicas tendem a residir em espaços urbanos que apresentam melhores condições estéticas, físicas e de segurança (Hofelmann et al., 2013)

Nos dados da área central da cidade de São Paulo, chama à atenção a grande sensação de insegurança referida pelos moradores dos dois grupos. Praticamente não há diferenças importantes entre os grupos com relação

\section{Vizinhança com vulnerabilidade}

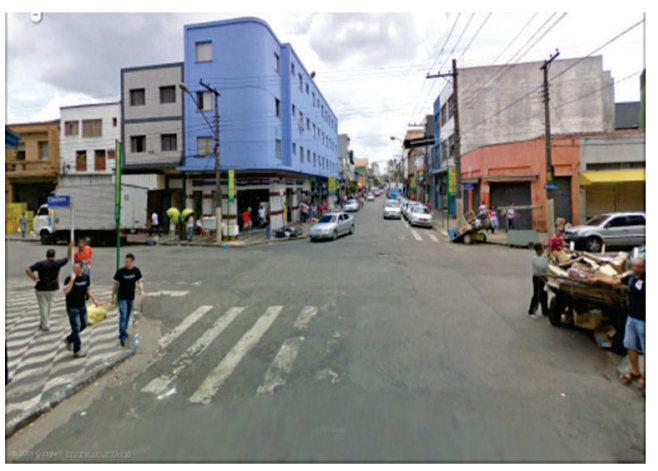

As vizinhanças em áreas vulneráveis são pouco ou nada arborizadas. Espaços verdes são menos frequentes e em pior estado de conservação. As calçadas e as ruas são mais degradadas.

Há lixo na rua e as edificações mais simples apresentam um estado razoável de conservação.

Moradores em situação de rua, profissionais do sexo e usuários de drogas são compõem um contingente importante de excluídos em uma área vulnerável.

Pichações são frequentes nessa paisagem urbana e degradada. 
à sensação de segurança para andar à noite pela vizinhança e ao medo de assalto. Embora este seja mais intenso no grupo socialmente vulnerável, também é bastante expressivo no grupo não vulnerável. Assim, como muitos autores mencionam, a percepção das condições de vizinhança deve ser considerada além das características materiais do ambiente construído, já que aparentemente mensura dimensões diferentes (ibid.).

\section{Atividade física: vulnerabilidade e vizinhança}

Inatividade, obesidade, tabagismo e consumo de álcool estão entre os fatores de risco relacionados aos comportamentos e estilos de vida que participam da determinação social das doenças crônicas e problemas de saúde mental (Zanchetta, 2010).

Atividade física é um comportamento que envolve diferentes atividades executadas no ambiente de trabalho, no trajeto para o trabalho ou na realização de serviços domésticos e também no lazer. Combinando a intensidade e a duração dos diferentes tipos de atividades físicas, é possível classificar os indivíduos em inativos, pouco ativos e ativos.

No cotidiano das grandes cidades, a adoção de um estilo de vida ativo não depende apenas do conhecimento sobre os benefícios que a atividade física pode trazer, mas principalmente das condições concretas para sua prática por diferentes grupos sociais. A ausência de equipamentos e espaços sociais para a prática de esportes e exercícios físicos, poluição sonora e atmosférica, excesso de tráfego de veículos motorizados, degradação dos entornos físicos, violência urbana e sensação de insegurança são aspectos que podem reduzir a proporção de adultos ativos (Jongennel-Grimen et al., 2014; Fernandes et al., 2015; Johnson-Lawrence et al., 2015).

Se considerarmos o ambiente urbano de grandes cidades como um determinante estrutural no processo saúde-doença e a posição social dos indivíduos (vulnerabilidade social) e o espaço socialmente construído onde habitam (vizinhança) como determinantes intermediários que condicionam o cotidiano dos grupos e dos indivíduos que os compõem, a atividade física pode ser pensada como um determinante proximal, decorrente do comportamento em relação à saúde moldado pelos processos sociais de nível intermediário e pela macroestrutura.

$\mathrm{Na}$ amostra estudada pelos autores, havia 25,6\% (IC95\%: 22,9 - 28,5) de adultos inativos, 41,1\% (IC95\%: 38,0 - 44,3) de adultos pouco ativos e 33,3\% (IC95\%: 30,3-36,4) de adultos ativos. No inquérito de saúde de adultos da Califórnia (Meyer; Castro-Schilo e Aguilar-Gaxiola, 2014), em 2009, a prevalência de pessoas ativas $(20,0 \%)$ foi menor do que a observada na amostra do centro de São Paulo, a proporção de pessoas pouco ativas foi semelhante $(44,0 \%)$ e a de inativos foi mais alta $(36,0 \%)$.

0 nível de atividade física foi medido por meio da aplicação International Physical Activity Questionnaire (Ipaq) versão curta, que indaga sobre as atividades realizadas na última semana, levando-se em consideração o tempo e a intensidade.

Os moradores da área central da cidade de São Paulo mostram distribuição diferente do grau de atividade física e também na composição dessa atividade entre trabalho ou lazer. 
Tabela 3 - Grau de atividade física, atividade física no trabalho e ter carro para moradores da área central da cidade de São Paulo, grupo sem ou com vulnerabilidade social, 2008

\begin{tabular}{l|c|c|c}
\multicolumn{1}{c|}{ Atividade física } & RP* (IC95\%) & Grupo sem vulnerabilidade & Grupo com vulnerabilidade \\
\hline $\begin{array}{l}\text { Grau de atividade } \\
\text { Inativo }\end{array}$ & $0,5(0,4-0,6)$ & $34,4(30,0-39,0)$ & $17,9(14,7-21,5)$ \\
Pouco ativo & $0,8(0,7-1,0)$ & $44,2(39,5-48,9)$ & $38,4(34,1-42,8)$ \\
Ativo & $2,0(1,6-2,6)$ & $21,4(17,7-25,5)$ & $43,7(39,4-48,2)$ \\
\hline No trabalho & & & $20,7(17,3-24,5)$ \\
Não & $0,3(0,2-0,4)$ & $59,5(54,8-64,1)$ & $79,3(75,5-82,7)$ \\
Sim & $1,9(1,6-2,3)$ & $40,5(35,9-45,2)$ & \\
\hline Carro & & & $76,9(73,0-80,5)$ \\
Nenhum & $2,1(1,8-2,6)$ & $35,5(31,1-40,1)$ & $19,0(15,7-22,6)$ \\
Um & $0,4(03-0,5)$ & $47,9(43,2-52,6)$ & $4,1(2,6-6,2)$ \\
Dois ou mais & $0,2(0,1-0,4)$ & $16,6(13,3-203)$ & \\
\hline
\end{tabular}

* Razão de prevalência.

A posse de carro pode reduzir a atividade física nos deslocamentos ativos e também apresenta distribuição desigual entre os grupos.

A razão de prevalência permite comparar as duas distribuições considerando o grupo sem vulnerabilidade social como o grupo de referência, ou seja, aquele não exposto a condições sociais adversas. É possível observar que o grupo com vulnerabilidade social apresentou menor probabilidade de ter indivíduos inativos (valor menor que 1,0 ) e maior probabilidade de ter indivíduos ativos (valor maior que 1,0 ). Quanto aos indivíduos pouco ativos, eles tiveram proporção semelhante nos dois grupos (diferenças não significativas).

Os resultados também mostram que 0 grupo com vulnerabilidade social tem maior probabilidade de ser ativo no trabalho e/ou no trajeto para ele, uma vez que foi mais provável para esses indivíduos não terem carro.

Os dados parecem contradizer a associação esperada entre melhores condições sociais, melhor vizinhança e maior atividade física, no entanto é preciso considerar que o grupo sem vulnerabilidade social tinha distribuição etária com menor proporção de jovens e maior proporção de idosos, o que afeta a distribuição do grau de atividade.

Dos adultos entrevistados, $72 \%$ disseram estar satisfeitos com as condições do bairro em que moravam. No entanto, $42 \%$ mencionaram que as ruas são pouco iluminadas, $60 \%$ afirmaram haver muito barulho, $86 \%$ poluição do ar, $58 \%$ presença de lixo ou entulho nas ruas e $62 \%$ consideram que faltam parques, jardins e outros espaços verdes em sua vizinhança. Quanto à percepção de aspectos relativos à segurança, $81 \%$ acreditam que as ruas e calçadas oferecem riscos para os pedestres, $76 \%$ sentem-se inseguros de andar à noite na vizinhança $e$ $56 \%$ têm frequentemente medo de ter a casa assaltada. Desse modo, as condições na área estudada parecem pouco propícias às atividades físicas no lazer em sua própria vizinhança. 
Tabela 4 - Prevalência de atividade física segundo características da vizinhança de moradores da área central da cidade de São Paulo, 2008

\begin{tabular}{|c|c|c|c|}
\hline Vizinhança & Inativo & Pouco ativo & Ativo \\
\hline $\begin{array}{l}\text { Satisfação com o bairro } \\
\text { Insatisfeito } \\
\text { Satisfeito } \\
\text { Muito satisfeito }\end{array}$ & $\begin{array}{r}15,8(9,4-24,1) \\
26,6(23,3-30,1) \\
26,7(20,3-33,9)\end{array}$ & $\begin{array}{l}36,8(27,6-46,8) \\
43,0(39,3-46,8) \\
36,6(29,5-44,3)\end{array}$ & $\begin{array}{l}47,4(37,5-57,4) \\
30,4(26,9-339) \\
36,6(29,5-44,3)\end{array}$ \\
\hline $\begin{array}{l}\text { Ruas pouco iluminadas } \\
\text { Não } \\
\text { Sim }\end{array}$ & $\begin{array}{l}29,4(25,6-33,4) \\
19,9(16,2-24,3)\end{array}$ & $\begin{array}{l}40,7(36,6-45,0) \\
42,0(37,1-46,9)\end{array}$ & $\begin{array}{l}29,9(26,1-33,9) \\
38,1(33,3-43,0)\end{array}$ \\
\hline $\begin{array}{l}\text { Há muito barulho } \\
\text { Não } \\
\text { Sim }\end{array}$ & $\begin{array}{l}29,9(25,4-34,8) \\
22,8(19,5-26,5)\end{array}$ & $\begin{array}{l}37,6(32,8-42,7) \\
43,5(39,4-47,6)\end{array}$ & $\begin{array}{l}32,4(27,7-37,4) \\
33,7(29,8-37,7)\end{array}$ \\
\hline $\begin{array}{l}\text { Há poluição ambiental } \\
\text { Não } \\
\text { Sim }\end{array}$ & $\begin{array}{l}26,4(19,3-34,5) \\
25,4(22,5-28,6)\end{array}$ & $\begin{array}{l}41,9(33,6-50,5) \\
41,0(37,6-44,4)\end{array}$ & $\begin{array}{l}31,8(24,2-40,2) \\
33,6(30,4-37,0)\end{array}$ \\
\hline $\begin{array}{l}\text { Há lixo e entulho nas ruas } \\
\text { Não } \\
\text { Sim }\end{array}$ & $\begin{array}{l}32,0(27,5-36,8) \\
21,2(17,9-24,8)\end{array}$ & $\begin{array}{l}36,2(31,5-41,1) \\
44,8(40,4-48,9)\end{array}$ & $\begin{array}{l}31,8(27,2-36,5) \\
34,3(30,3-38,4)\end{array}$ \\
\hline $\begin{array}{l}\text { Faltam espaços verdes } \\
\text { Não } \\
\text { Sim }\end{array}$ & $\begin{array}{l}30,5(25,8-35,5) \\
22,7(19,4-26,3)\end{array}$ & $\begin{array}{l}43,0(37,8-48,3) \\
39,9(35,9-43,9)\end{array}$ & $\begin{array}{l}26,5(22,0-31,3) \\
37,4(33,5-41,4)\end{array}$ \\
\hline $\begin{array}{l}\text { Há riscos para pedestres e/ou ciclistas } \\
\text { Não } \\
\text { Sim }\end{array}$ & $\begin{array}{l}24,0(18,1-30,7) \\
26,0(22,9-29,2)\end{array}$ & $\begin{array}{l}41,1(34,0-48,5) \\
41,0(37,5-44,5)\end{array}$ & $\begin{array}{l}34,9(28,1-42,1) \\
33,0(29,7-36,5)\end{array}$ \\
\hline $\begin{array}{l}\text { Caminhar à noite dá sensação de: } \\
\text { Muita segurança } \\
\text { Segurança } \\
\text { Insegurança }\end{array}$ & $\begin{array}{r}22,5(11,6-37,3) \\
14,1(9,5-19,8) \\
28,4(25,1-31,8) \\
\end{array}$ & $\begin{array}{l}37,5(23,6-53,1) \\
46,9(39,6-54,3) \\
40,1(36,5-43,7) \\
\end{array}$ & $\begin{array}{l}40,0(25,8-55,6) \\
39,0(32,0-46,3) \\
31,6(28,2-35,1)\end{array}$ \\
\hline $\begin{array}{l}\text { Medo de assalto } \\
\text { Todo o tempo } \\
\text { Frequentemente } \\
\text { Nunca }\end{array}$ & $\begin{array}{l}16,0(10,3-23,2) \\
31,2(27,3-35,4) \\
19,9(15,5-24,8)\end{array}$ & $\begin{array}{l}47,2(38,6-56,0) \\
41,8(37,6-46,2) \\
37,2(31,7-42,9)\end{array}$ & $\begin{array}{l}36,8(28,7-45,5) \\
26,9(23,2-30,9) \\
42,9(37,2-48,7)\end{array}$ \\
\hline
\end{tabular}

Alguns resultados parecem contradizer o senso comum, no entanto é preciso considerar que predominam condições insatisfatórias, e parte considerável da atividade física, nessa população, relaciona-se com o trabalho e não com o lazer. Assim, a satisfação com a vizinhança não parece estar associada ao nível de atividade física, provavelmente porque a satisfação pode estar referida a outros aspectos que não as condições físicas do entorno. A falta de iluminação, o barulho, a poluição do ar e a presença de lixo nas ruas não parecem alterar a distribuição dos níveis de atividade dos indivíduos. Entretanto, os dados não permitem avaliar se haveria maior proporção de pessoas ativas caso as condições de vizinhança fossem mais favoráveis. Coerentemente, os mais ativos foram aqueles que mais perceberam a falta de espaços verdes. Como a maioria dos entrevistados considerou que as ruas são pouco seguras 
para os pedestres, essa percepção não parece alterar a distribuição dos níveis de atividade física. Novamente, não foi possível avaliar se a proporção de ativos seria maior em condições de menor risco. Em San Diego, Califórnia, autores observaram que a percepção de segurança ou o risco para os pedestres foi menos relevante para determinar os níveis de atividade física do que as condições das calçadas (Bracy et al., 2014). Em Chicago, para os participantes do estudo multiétnico de aterosclerose (Mesa), os autores observaram que alterações na percepção de segurança ou nos registros policiais sobre crimes não estavam associadas com 0 transporte ativo ou atividade física no lazer, exceto em áreas em que houve aumento de assassinatos, nas quais foram observados grandes declínios no deslocamento ativo dos moradores (Kerr et al., 2015). Finalmente, a percepção de insegurança para andar à noite e o medo frequente de assalto parecem reduzir de modo significativo entre a proporção de ativos, ou as pessoas mais ativas tendem a perceber sua vizinhança como segura.

Resultados semelhantes foram observados em Detroit (Kwarteng et al., 2013), onde apenas as condições das calçadas mostraram associação com o nível de atividade física. Em Calgary (Jack e MCCormack, 2014), os pesquisadores observaram que há boa correspondência entre as informações dos entrevistados e a observação direta sobre as condições de vizinhança. Entretanto, notaram maior influência dessas condições sobre o transporte ativo do que sobre as atividades físicas no lazer, principalmente no que se refere à duração. Como no estudo realizado em São Paulo, o nível de atividade física foi menos influenciado pelas características físicas de vizinhança exatamente naquelas consideradas mais insatisfatórias, sugerindo que a necessidade de deslocamento, provavelmente para o trabalho, impõe-se aos grupos com maior vulnerabilidade social. Em locais em que as condições são melhores, a sensação de insegurança desempenha um papel importante na redução da prática da atividade física.

Em Curitiba, analisando apenas atividade física no lazer e caminhadas em particular, os autores encontraram associação entre os níveis de atividade física no lazer e a sensação de segurança, aspectos estéticos da vizinhança e acesso a espaços como parques e jardins (Rech et al., 2014).

Estudo multicêntrico envolvendo 16 cidades em 11 países, incluindo Curitiba no Brasil, encontrou grande variabilidade nos níveis de atividade física em modelos ajustados e não ajustados para as características de vizinhança. A variável que mostrou a maior correlação com níveis satisfatórios de atividade física foi o uso misto, residencial e comercial, do espaço construído. A sensação de segurança na vizinhança foi mais importante para alcançar níveis mais altos de atividade física. Os aspectos estéticos como encorajadores da prática de atividade física são particularmente importantes em cidades norte-americanas (ibid.)

Tendo em vista que, na área central da cidade de São Paulo, as condições de vizinhança são distintas nos setores censitários sem vulnerabilidade e naqueles classificados como tendo média, alta ou altíssima vulnerabilidade social e que os resultados observados em diferentes cidade e grupos populacionais são muito variáveis, é provável que a mediação exercida pelas condições de vizinhança sobre os níveis de atividade física varie 
para esses diferentes grupos populacionais (Boclin, Faerstein e Leon, 2014). Além disso, é importante lembrar que, nos setores sem vulnerabilidade, a atividade física distribuía-se igualmente entre atividade no trabalho e no transporte ativo, por um lado, e lazer, por outro; enquanto nos setores vulneráveis apenas um quarto das atividades físicas estavam associadas ao lazer. Resultados semelhantes foram encontrados no estudo Pró-Saúde em funcionários de uma universidade pública carioca (ibid.). Algumas das características de vizinhança, como a percepção ou não de poluição atmosférica e de risco para pedestres e ciclistas, não mostraram associação com os níveis de atividade física em ambos os grupos. Ou seja, tanto nos setores sem vulnerabilidade social quanto naquele com vulnerabilidade social, a distribuição dos níveis de atividade física não foi afetada.

Tabela 5 - Nível de atividade física segundo características de vizinhança para grupo sem ou com vulnerabilidade social na área central da cidade de São Paulo, 2008

\begin{tabular}{|c|c|c|c|}
\hline Vulnerabilidade e vizinhança & Inativo & Pouco ativo & Ativo \\
\hline \multicolumn{4}{|l|}{ Sem vulnerabilidade } \\
\hline $\begin{array}{l}\text { Satisfação com o bairro } \\
\text { Insatisfeito* } \\
\text { Satisfeito } \\
\text { Muito satisfeito }\end{array}$ & $\begin{array}{c}33.3 \\
36.9(31.7-42.3) \\
27.1(19.3-36.1)\end{array}$ & $\begin{array}{c}50.0 \\
44.8(39.4-50.3) \\
42.1(32.9-51.5)\end{array}$ & $\begin{array}{c}16.7 \\
18.5(14.3-22.8) \\
30.8(22.6-40.1)\end{array}$ \\
\hline $\begin{array}{l}\text { Ruas pouco iluminadas } \\
\text { Não } \\
\text { Sim }\end{array}$ & $\begin{array}{l}39.0(33.7-44.4) \\
20.7(13.9-29.0)\end{array}$ & $\begin{array}{l}41.2(35.9-46.7) \\
53.2(43.9-62.3)\end{array}$ & $\begin{array}{l}19.8(15.7-24.5) \\
26.1(18.6-34.8)\end{array}$ \\
\hline $\begin{array}{l}\text { Lixo e entulho nas ruas } \\
\text { Não } \\
\text { Sim }\end{array}$ & $\begin{array}{l}38.4(32.5-44.4) \\
28.7(22.3-35.8)\end{array}$ & $\begin{array}{l}38.0(32.2-44.0) \\
53.8(46.3-61.2)\end{array}$ & $\begin{array}{l}23.6(18.7-29.1) \\
17.5(12.4-23.8)\end{array}$ \\
\hline $\begin{array}{l}\text { Caminhar à noite dá sensação de: } \\
\text { Muita segurança* } \\
\text { Segurança } \\
\text { Insegurança }\end{array}$ & $\begin{array}{c}35.0 \\
17.6(10.1-27.5) \\
38.0(32.9-43.3)\end{array}$ & $\begin{array}{c}40.0 \\
52.7(41.3-63.8) \\
42.5(37.2-47.8)\end{array}$ & $\begin{array}{c}25.0 \\
29.7(20.1-40.8) \\
19.5(15.5-23.9)\end{array}$ \\
\hline $\begin{array}{l}\text { Medo de assalto } \\
\text { Todo o tempo* } \\
\text { Frequentemente } \\
\text { Nunca }\end{array}$ & $\begin{array}{c}28.0 \\
39.9(34.4-45.6) \\
21.6(14.7-30.0)\end{array}$ & $\begin{array}{c}64.0 \\
42.3(36.8-48.0) \\
45.0(36.0-54.3)\end{array}$ & $\begin{array}{c}8.0 \\
17.7(13.7-22.4) \\
33.3(25.0-42.4)\end{array}$ \\
\hline Com vulnerabilidade & & & \\
\hline $\begin{array}{l}\text { Há muito barulho } \\
\text { Não } \\
\text { Sim }\end{array}$ & $\begin{array}{r}25,0(19.5-31.1) \\
12,4(8.9-16.7)\end{array}$ & $\begin{array}{l}33,5(27.3-40.1) \\
42,3(36.6-48.2)\end{array}$ & $\begin{array}{l}41,5(35.0-48.2) \\
45,3(39.4-51.2)\end{array}$ \\
\hline $\begin{array}{l}\text { Faltam espaços verdes } \\
\text { Não } \\
\text { Sim }\end{array}$ & $\begin{array}{l}21,9(15.6-29.4) \\
16,3(12.7-20.5)\end{array}$ & $\begin{array}{l}40.9(32.8-49.3) \\
37,2(32.3-42.4)\end{array}$ & $\begin{array}{l}37.2(29.4-45.5) \\
46,4(41.2-51.7)\end{array}$ \\
\hline
\end{tabular}

* número muito pequeno. 
Outras características mostraram associação em apenas um dos grupos. A maioria delas não alterou a distribuição dos níveis de atividade física nos setores censitários vulneráveis, enquanto várias foram relevantes nos setores censitários não vulneráveis.

Os resultados do inquérito de saúde da Califórnia, em população adulta, reforçaram a hipótese de que altos níveis socioeconômicos estavam associados com maior sensação de segurança e com maior atividade física no lazer, que, por sua vez, estava associada a meIhor estado de saúde autorreferido e melhor saúde mental. Os autores verificaram, ainda, que o modelo foi válido independentemente da idade, do gênero e da etnia. No nosso estudo, considerando a atividade física total, os maiores níveis foram observados para os grupos socialmente vulneráveis, e a associação com sensação de segurança só foi significativa no grupo não vulnerável (Meyer, Castro-Schilo e Aguilar-Gaxiola, 2014).

A satisfação com a vizinhança só esteve associada aos níveis de atividade física nos setores censitários sem vulnerabilidade social. Como poucos indivíduos disseram estar insatisfeitos com seu bairro, as diferenças foram observadas para aqueles satisfeitos versus os muito satisfeitos. Os indivíduos pouco ativos foram a categoria mais frequente em ambos os subgrupos, e a diferença entre eles ocorreu em relação aos ativos que foram mais frequentes entre os muito satisfeitos ( $R P=1,69$ IC95\%:1,09-2,58),

Quanto à iluminação das ruas, o resultado foi paradoxal, sugerindo a interferência de fatores de confusão. Não houve diferença significante para pouco ativos e ativos segundo iluminação das ruas, e observou-se proporção menor de inativos justamente para os que referiram considerar as ruas pouco iluminadas ( $\mathrm{RP}=0.53$ IC95\%: 0.33-0.82). Esse resultado poderia também estar indicando que justamente os indivíduos mais ativos valorizam mais a questão da iluminação das ruas.

A presença de lixo e entulho nas ruas esteve associado apenas no caso das pessoas pouco ativas, em que a proporção foi maior exatamente nos locais com maior presença de lixo e entulho nas ruas. Para os ativos e os inativos, as diferenças não foram significativas.

A sensação de segurança para caminhar à noite pelo bairro influenciou a proporção de inativos na direção esperada, isto é, a proporção de inativos foi maior nas áreas consideradas mais inseguras ( $\mathrm{RP}=2,16 \mathrm{IC} 95 \%: 1,26$ $-3,99)$. Para as pessoas ativas, observou-se distribuição contrária, porém, dado o pequeno número delas, a razão de prevalência não foi significativa. Estudo realizado em Cuernavaca, no México, no qual os setores censitários foram estratificados em oito grupos de acordo com dados socioeconômicos e condições favoráveis à caminhada, os autores observaram que o nível de atividade física referente ao deslocamento ativo não se associou à percepção de segurança do bairro. Entretanto, como a amostra era pequena, os dados devem ser analisados com cautela (Salvo et al., 2014).

Com relação ao medo de assalto, observou-se maior proporção de inativos nas áreas em que os indivíduos têm frequentemente medo de assaltos ( $R P=1,85$ IC95\%:1,21 - 2,92) e maior proporção de ativos nas áreas em que os indivíduos referiam nunca ter medo de assaltos ( $R P=1,87$ IC95\%:1,22-2,86). A chance de ter indivíduos inativos foi 1,85 vezes maior nas áreas em que eles têm medo de assaltos 
enquanto a chance de ter indivíduos ativos foi 1,87 vezes maior nas áreas em que eles nunca têm medo.

Para os setores censitários com vulnerabilidade social, apenas dois aspectos estiveram associados com o nível de atividade física: ruído e falta de espaços de lazer, como parques e jardins. A referência a excesso de barulho, principalmente relacionado ao tráfego de veículos, associou-se com a proporção de inativos, mas na direção contrária ao esperado, uma vez que foram observados mais inativos nas áreas em que o ruído não foi considerado um problema. Para os pouco ativos e ativos não houve diferença significativa quanto ao ruído.

Com relação à falta de espaços para a prática de atividades físicas no lazer, como parques, jardins e outros, a maioria reconheceu a escassez desses espaços no bairro. A diferença entre aqueles que perceberam essa falta e os demais não foi significativa para nenhum dos níveis de atividade física.

Em inquérito realizado com população adulta de três cidades norte-americanas, a única associação significativa encontrada foi entre percepção de segurança para pedestres e transporte ativo. No entanto, tal associação só foi verificada para indivíduos com maior grau de escolaridade e maior renda (Carlson, 2014).

Em amostra de mulheres em idade reprodutiva, residentes em áreas socialmente vulneráveis, no estado de Victoria na Austrália, os autores encontraram $44,2 \%$ de mulheres ativas, valor semelhante ao observado em São Paulo para o grupo vulnerável. 0 nível de atividade física nesse grupo se associou positivamente com a percepção de segurança pessoal no bairro e com o nível de confiança nos vizinhos. Outra variável que se mostrou positivamente associada foi a observação de outros moradores caminhando na área (Timperio, Veitch e Carver, 2015).

Como muitas das características de vizinhança apresentam alguma correlação entre si e a atividade física pode sofrer a influência de características sociodemográficas dos moradores além das influências contextuais da vizinhança e da vulnerabilidade social, os resultados foram modelados através de uma regressão nominal, a fim de identificar a importância das diferentes variáveis sobre o nível de atividade física.

Para os moradores nos setores censitários vulneráveis, o nível de atividade física só mostrou associação com idade, nível de ruído e falta de espaços verdes na vizinhança. Apenas entre os idosos observou-se menor proporção de ativos e maior de inativos, mas a amostra era constituída por apenas 44 idosos, número insuficiente para a modelagem. Quanto à associação com ruído, ela foi contraditória havendo menos inativos nas áreas mais barulhentas. $A$ percepção da falta de espaços verdes, embora significativa em termos de ocorrência, não se mostrou significante na força de associação. Os autores optaram então por não fazer a modelagem para esse grupo.

Para os moradores nos setores censitários não socialmente vulneráveis, os níveis de atividade física foram ajustados por idade, gênero, escolaridade, nível de consumo, ter carro, renda familiar e características de vizinhança, exceto ruído, poluição e espaços verdes que não mostraram associação na análise bivariada.

Após o ajuste realizado através do modelo de regressão, as variáveis que se associaram com o nível de atividade física foram identificadas para pessoas pouco ativas e pessoas ativas. 
Tabela 6 - Razão de chances e intervalo de confiança de $95 \%$ para a associação entre características sociodemográficas e vizinhança e nível de atividade física para moradores dos setores censitários sem vulnerabilidade social obtidos por meio de Regressão Logística Multinomial*

\begin{tabular}{l|c|c}
\multicolumn{1}{c|}{ Variáveis } & Odds Ratio & IC95\% \\
\hline Pouco Ativo & & \\
Homens & 2,90 & $1,78-4,72$ \\
Sem carro & 5,52 & $2,56-11,91$ \\
Sensação de segurança à noite & 3,14 & $1,48-6,67$ \\
\hline Ativo & & $1,58-5,15$ \\
Homens & 2,85 & $2,73-17,17$ \\
Sem carro & 6,85 & $1,11-6,14$ \\
Sensação de segurança à noite & 2,62 & \\
\hline
\end{tabular}

* Regressão Logística Multinomial é uma técnica estatística utilizada em análise multivariadas para investigar a associação de um conjunto de variáveis independentes e uma variável desfecho com três ou mais categorias. No caso, a variável desfecho é o nível de atividade física e a categoria inativo foi utilizada como referência.

Portanto, das variáveis analisadas, após o ajuste para as demais, foram associadas positivamente com o nível de atividade física: ser homem, não ter carro e se sentir seguro para andar à noite em sua vizinhança. Não mostraram associação a idade, a escolaridade, a renda familiar, o nível de consumo e as características físicas da vizinhança. As mesmas variáveis aumentaram a chance de ser pouco ativo em comparação com os inativos ou de ser ativo, isto é, alcançar os níveis de atividade física considerados mínimos para produzir impactos positivos sobre a saúde em geral.

Em Pelotas, os autores identificaram como mais ativos no lazer os homens com maior nível de escolaridade, renda mais alta e residentes na área central da cidade. Com relação ao transporte ativo, os autores encontraram como mais ativos os homens mais jovens e com menor renda média. As variáveis relativas à percepção de insegurança no bairro de residência (crimes, roubos, tráfico de drogas e homicídios) não mostraram associação com nenhum dos tipos de atividade física (Mendes et al., 2014).

As relações entre nível socioeconômico, características de vizinhança e atividade física são bastante complexas e difíceis de serem apreendidas adequadamente. As motivações para a prática de atividade física e as condições objetivas para seu exercício são distintas nos diferentes grupos sociais. Entre a população mais vulnerável, a maior parte da atividade física está relacionada com o trabalho. Esse fato tem duas implicações importantes: primeiro, ao considerar em inquéritos populacionais, realizados em países de média ou baixa renda, a taxa de pessoas inativas, pouco ativas ou ativas, sem separar os diferentes grupos sociais, as medidas tenderão a estar superestimadas, uma vez que a maioria da população pertence aos grupos com piores condições socioeconômicas; 
segundo, a atividade física despendida no trabalho parece desestimular a prática de atividade física no lazer, como uma espécie de mecanismo de compensação.

Assim, para os grupos mais vulneráveis, as características físicas da vizinhança parecem não ter importância no estímulo ou desestímulo de atividade física no lazer, com exceção talvez do grupo mais jovem.

Para os grupos com melhores condições socioeconômicas, a atividade física total é dividida entre atividade física no trabalho e no lazer, podendo haver maior influência do contexto de vizinhança sobre a atividade no lazer. No entanto, esse grupo, justamente por sua situação econômica, pode fazer uso de outros recursos, tais como clubes, parques e outros espaços de lazer que não necessariamente estejam localizados no bairro de residência. Em um município como São Paulo, com alta densidade de edificações nos bairros situados na área central, é menos provável a existência desses equipamentos públicos ou privados no bairro de residência.
Por fim, é preciso ainda considerar as limitações próprias de estudos transversais desenvolvidos por meio de inquérito domiciliar. Um aspecto é o chamado viés de sobrevivência, ou seja, apenas indivíduos não institucionalizados e vivos, no momento da pesquisa, podem ser alcançados pelas entrevistas. Essa seleção poderia subestimar as associações estudadas. Outro aspecto foi a taxa de recusa maior entre as pessoas com melhores condições de vida. 0 processo de amostragem buscou minimizar esse efeito. Houve, contudo, menos entrevistas em estratos sem vulnerabilidade social. Acreditamos, no entanto, que a comparação entre os estratos não deve ter sido influenciada por esse aspecto, diferentemente do que ocorreria na produção de uma estimativa geral.

De todo modo, é importante considerar, quando se trata de saúde urbana, a complexidade dos processos sociais e ambientais que podem contribuir para a manutenção do estado de saúde ou para a produção das doenças nos diferentes grupos sociais que compartiIham o espaço urbano.

\section{Manoel Carlos Sampaio de Almeida Ribeiro}

Faculdade de Ciências Médicas da Santa Casa de São Paulo, Departamento de Saúde Coletiva. São Paulo, SP/Brasil.

mcrmacal@gmail.com

\section{Rita Barradas Barata}

Faculdade de Ciências Médicas da Santa Casa de São Paulo, Departamento de Saúde Coletiva. São Paulo, SP/Brasil.

rita.barradasbarata@gmail.com 


\section{Referências}

BARATA, R. B.; RIBEIRO, M. C. S. A. e CASSANTI, A. C. (2011). Social vulnerability and health status: a household survey in the central area of a Brazilian metropolis. Cadernos de Saúde Pública. Rio de Janeiro, v. 27, sup. 2, pp. S164-S175

BOCLIN, K. L. S.; FAERSTEIN, E. e LEON, A. C. M. P. (2014). Neighborhood contextual characteristics and leisure-time physical activity: Pró-Saúde Study. Revista de Saúde Pública. São Paulo, v. 48, n. 2, pp. 249-257.

BOONE-HEINONEN, J.; DIEZ-ROUX, A. V.; KIEFE, C. I.; LEWIS, C. E.; GUILKEY, D. K. e GORDON-LARSEN, P. (2011). Neighborhood socioeconomic status predictors of physical activity thrugh young to mild adulthood: the cardia study. Social Science \& Medicine. Oxford, v. 72, n. 5, pp. 641-649.

BOUSQUAT, A. e NASCIMENTO, V. B. (2001). A metrópole paulista e a saúde. São Paulo em Perspectiva. São Paulo, v. 15, n. 1, pp. 112-120.

BRACY, N.; MILLSTEIN, R. A.; CARLSON, J. A.; CONWAY, T. L.; SALLIS, J. F.; SAELENS, B. E.; KERR, J.; CAIN, K. L.; FRANK, L. D. e KING, A. C. (2014). Is the relationship between the built environment and physical activity moderated by perceptions of crime and safety? International Journal of Behavioral Nutrition and Physical Activity. Londres, v. 11, p. 24.

CARLSON, J. A.; BRACY, N. L.; SALLIS, J. F.; MILLSTEIN, R. A.; SAELENS, B. E.; KERR, J.; CONWAY, T. L.; FRANK, L. D.; CAIN, K. L. e KING, A. C. (2014). Sociodemographic moderators of relations of neighborhood safety to physical activity. Medicine and Science in Sports and Exercise. Madison, v. 46, n. 8 , pp. $1554-1563$.

CASTELLANOS, P. L. (1997). “Epidemiologia, saúde pública, situação de saúde e condições de vida: considerações conceituais". In: BARATA, R. B. (org.). Condições de vida e situação de saúde. Rio de Janeiro, Abrasco.

CERIN, E.; CAIN, K. L.; CONWAY, T. L.; VAN DYCK, D.; HINCKSON, E.; SCHIPPERIJN, J.; BOURDEAUDHUIJ, I. D.; OWEN, N.; DAVEY, R. C.; HINO, A. A. F.; MITAS, J.; ORZANCO-GARRALDA, R.; SALVO, D.; SARMIENTO, O. L.; CHRISTIANSEN, L. B.; MACFARLANE, D.; SCHOFIELD, G. e SALLIS, J. F. (2014). Neighborhood environments and objectively measured physical activity in 11 countries. Medicine and Science in Sports and Exercise. Madison, v. 46, n. 12, pp. 2253-2264.

FERnANDES, A. P.; ANDRADE, A. C. S.; RAMOS, C. G. C.; FRICHE, A. A. L.; DIAS, M. A. S.; XAVIER, C. C.; PROIETTI, F. A. e CAIAFFA, W. T. (2015). Atividade física de lazer no território das Academias da Cidade, Belo Horizonte, Minas Gerais, Brasil: o efeito da presença de um programa de promoção da saúde na comunidade. Cadernos de Saúde Pública. Rio de Janeiro, v. 31, sup, pp. S1-S13.

FUNDAÇÃO SEADE (2010). Índice paulista de vulnerabilidade social. Disponível em: http://www. indices-ilp.al.sp.gov.br. Acesso em: mar 2010.

(2015). Informações dos municípios paulistas. Perfil Municipal. Disponível em: http://www. imp.seade.gov.br. Acesso em: 11 nov 2015.

HOFELMANN, D. A.; DIEZ-ROUX, A. V.; ANTUNES, J. L. F. e PERES, M. A. (2013). Perceived neighborhood problems: multilevel analysis to evaluate psychometric properties in a Southern adult Brazilian population. BMC Public Health. Londres, v. 13, p. 1085.

IGLESIAS, W. (2002). Impactos da mundialização sobre uma metrópole periférica. Revista Brasileira de Ciências Sociais. São Paulo, v. 17, n. 50, pp. 47-180. 
JACK, E. e MCCORMACK, G. R. (2014). The associations between objectively-determined and selfreported urban form characteristics and neighborhood-based walking in adults. International Journal of Behavioral Nutrition and Physical Activity. Londres, v. 11, p. 71.

JOHNSON-LAWRENCE, V.; SCHULZ, A. J.; ZENK, S. N.; ISRAEL, B. A. e ROWE, Z. (2015). Does territoriality modify the relationship between perceived neighborhood challenges and physical activity? $A$ multilevel analysis. Annals of Epidemiology. Nova York, v. 25, pp. 107-112.

JONGENNEL-GRIMEN, B.; DROOMERS, M.; VAN OERS, H. A. M.; STRONKS, K. e KUNST, A. E. (2014). The relationship between physical activity and the living environment: a multi-level analyses focusing on changes over time in environmental factors. Health \& Place. Oxford, v. 26, pp. 149-160.

KERR, Z.; EVENSON, K. R.; MOORE, K.; BLOCK, R. e DIEZ-ROUX, A. V. (2015). Changes in walking associated with perceived neighborhood safety and police-record crime: the multi-ethnic study of atherosclerosis. Preventive Medicine. Nova York, v. 73, pp. 88-93.

KING, K. E. e CLARKE, P. J. (2015). A disadvantaged advantage in walkability: finfings from socioeconomic and geographical analysis of National Built Environment Data in the United States. American Journal of Epidemiology. Baltimore, v.181, n.1, pp. 17-25.

KWARTENG, J. L.; SCHULZ, A. J.; MENTZ, G. B.; ZENK, S. N. e OPPERMAN, A. A. (2013). Associations between observed neighborhood characteristics and physical activity: findings from a multiethnic urban community. Journal of Public Health. Oxford, v. 36, n. 3, pp. 358-367.

MENDES, M. A.; SAILVA, I. C. M.; HALLAL, P. C. e TOMASI, E. (2014). Physical activity and perceived insecurity from crime in adults: a population-based study. Plos One. San Francisco, v. 9, n. 9, pp. e108136.

MENG, S.; YA-YUN, T.; QING-MIN, L.; YAN-JUN, R.; KAWACHI, I.; LI-MING, L. e JUN, L. V. (2014). Association between perceived urban built environment atributes and leisure-time physical activity among adults in Hangzhou, China. International Journal of Behavioral Nutrition and Physical Activity. Londres, v. 11, p. 71.

MEYER, O. L.; CASTRO-SCHILO, L. e AGUILAR-GAXIOLA, S. (2014). Determinants of mental health and self-rated health: a modelo f socioeconomic status, neighborhood safety and physical activity. American Journal of Public Health. Nova York, v. 104, n. 9, pp. 1734-1741.

PAIM, J. S. (1997). “Abordagens teórico-conceituais em estudos de condições de vida e saúde: notas para reflexão em ação". In: BARATA, R. B. (org.). Condições de vida e situação de saúde. Rio de Janeiro, Abrasco.

PREFEITURA DO MUNICÍPIO DE SÃO PAULO (2011). Censo de população em situação de rua na municipalidade de São Paulo. Disponível em: http:// www.prefeitura.sp.gov.br Acesso em: 11 nov 2015.

RECH, C. R.; REIS, R. S.; HINO, A. A. F. e HALLAL, P. C. (2014). Personal, social and environmental correlates of physical activity in adults from Curitiba, Brazil. Preventive Medicine. Nova York, v. 58, pp. 53-57.

SALVO, D.; REIS, R. S.; STEIN, A. D.; RIVERA, J.; MARTORELL, R. e PRATT, M. (2014). Characteristics of the built environment in relation to objectively measured physical activity among Mexican adults, 2011. Preventing Chronic Diseases. Atlanta, v. 11, pp. 140047.

TIMPERIO, A.; VEITCH, J. e CARVER, A. (2015). Safety in numbers: does perceived safety mediate associations between the neighborhood social environmental and physical activity among women living in disadvantaged neighborhoods? Preventive Medicine. Nova York, v. 74, pp. 49-54. 
VLAHOV, D. (2015). A pivotal moment for urban health. Cadernos de Saúde Pública. Rio de Janeiro, v. 31 , sup. pp. S7-S8.

WORLD HEALTH ORGANIZATION. Commission on Social Determinants of Health (2007). A conceptual framework for action on the social determinants of health. Geneva.

ZANCHETTA, L. M.; BARROS, M. B. A.; CESAR, C. L. G.; CARANDINA, L.; GOLDBAUM, M. e ALVES, M. C.G. P. (2010). Inatividade física e fatores associados em adultos, São Paulo, Brasil. Revista Brasileira de Epidemiologia. São Paulo, v. 13, n. 3, pp. 387-399.

Texto recebido em 16/fev/2016

Texto aprovado em 6/abr/2016 